\title{
Maximizing the Spectral Brilliance \\ of an Undulator
}

L. C. Teng

October 1984

In an undulator with $N$ cells each having a length $\ell$ (total length $L \equiv N \ell$ ) and a sinusoidal transverse field

$$
B_{y}=B_{0} \sin \left(2 \pi \frac{s}{l}\right)
$$

where $s$ is the distance along the straight unperturbed orbit. - The undulated orbit is given by

$$
\left\{\begin{array}{l}
x^{\prime}=\frac{l}{2 \pi \rho_{0}} \cos \left(2 \pi \frac{s}{l}\right) \equiv \frac{K}{\gamma} \cos \left(2 \pi \frac{5}{l}\right) \\
x=\frac{l^{2}}{4 \pi^{2} \rho_{0}} \sin \left(2 \pi \frac{s}{l}\right) \equiv \frac{K^{2}}{\gamma^{2}} \rho_{0} \sin \left(2 \pi \frac{5}{l}\right)
\end{array}\right.
$$

where $\rho_{0} \equiv \frac{(B \rho)}{B}$ with $\left(B_{\rho}\right)=\frac{c p}{e}=$ rigidity of beam and where, so defined, $K \equiv \frac{\ell}{2 \pi \rho_{0}} / \frac{1}{\gamma}=$ (Beam angle)/(Radiation angle) is a measure of the degree of overlap of the synchrotron radiation from successive cells and characterizes therefore the nature of the device

$$
\begin{cases}K \gg 1 & \text { Wiggler } \\ K \approx 1 & \text { Undulator }\end{cases}
$$


The transition from one to the other is obviously not sharp and the formulas below apply to either device.

\section{A. Wave Length and Beam Size}

(The material in sections $A$ and $B$ can be found in published work such as S. Krinsky, IEEE Trans. on Nucl. Sci., Vol. NS-30, No. 4, p. 3078 (1983) and is reproduced here only for easy reference.)

The fundamental $(k=1)$ wave length of the radiation emitted at zero angle is just the excess distance travelled by the radiation when the beam has traversed one cell. This is

$$
\begin{aligned}
\lambda_{1} & =\ell\left(\frac{1}{\left\langle\beta \cos x^{\prime}\right\rangle}-1\right)=\ell\left\{\frac{1}{\beta\left[1-\frac{1}{2} \frac{K^{2}}{\gamma^{2}}\left\langle\cos ^{2}\left(2 \pi \frac{5}{\ell}\right)\right\rangle\right]}-1\right\} \\
& =\ell\left[\left(1+\frac{1}{2 \gamma^{2}}\right)\left(1+\frac{1}{4} \frac{K^{2}}{\gamma^{2}}\right)-1\right]=\frac{\ell}{2 \gamma^{2}}\left(1+\frac{K^{2}}{2}\right)
\end{aligned}
$$

This also is just the Doppler shifter radiation from the moving electrons. If, indeed, it were possible for the electrons to travel faster than light so that when they traversed one cell the radiation has travelled exactly a distance $\ell$ we would have $\lambda=0$ and the optical equivalent of the "sonic boom" which happens when the jet plane is travelling at the speed of sound at MACH 1. The harmonic wave lengths are

$$
\lambda_{k} \equiv \frac{\lambda_{1}}{k}=\frac{l}{2 k \gamma^{2}}\left(1+\frac{k^{2}}{2}\right)
$$

Only odd harmonics (odd k) are reinforced. 
For the radiation emitted at an angle $\theta$ the fundamental wave length is simply

$$
\begin{aligned}
\lambda_{1 \theta} & =\ell\left(\frac{1}{\left\langle\beta \cos x^{\prime}\right\rangle}-\cos \theta\right) \cong \frac{l}{2 \gamma^{2}}\left(1+\frac{k^{2}}{2}+\gamma^{2} \theta^{2}\right) \\
& =\lambda_{1}\left(1+\frac{\gamma^{2} \theta^{2}}{1+\frac{k^{2}}{2}}\right)
\end{aligned}
$$

For an $N$ cell undulator the diffraction resolution of $\lambda_{k}$ is $\frac{\Delta \lambda_{k}}{\lambda_{k}}=\frac{1}{k N}$. From Eq. (6) this is equivalent to an rms diffraction angle $\sigma_{r}$ 'defined by

$$
\frac{\Delta \lambda_{t}}{\lambda_{k}}=\frac{\gamma^{2}\left(2 \sigma_{r}^{\prime 2}\right)}{1+\frac{k^{2}}{2}}=\frac{1}{k N}
$$

or

$$
\sigma_{r}^{\prime 2}=\frac{1}{2 k N \gamma^{2}}\left(1+\frac{k^{2}}{2}\right)=\frac{\lambda k}{L}
$$

This result is physically quite transparent.

If the rms beam size and divergence at the midpoint of the undulator are $\sigma$ and $\sigma^{\prime}$ respectively, the apparent source size and divergence of the synchrotron radiation are clearly 


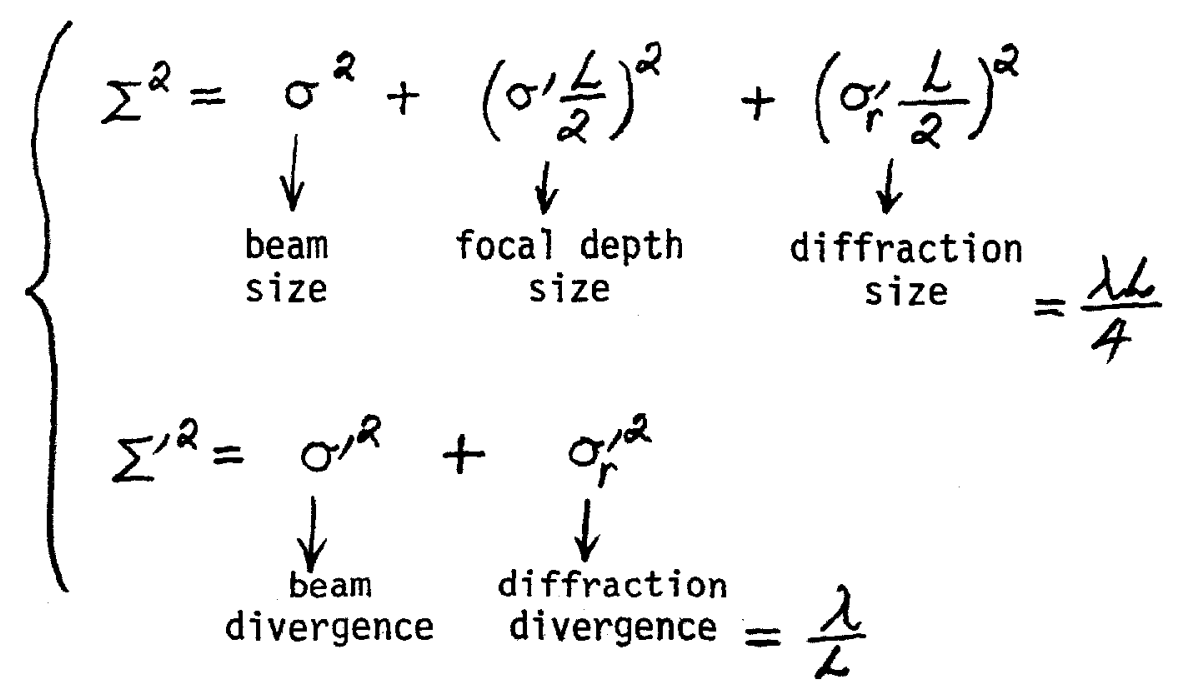

This is shown in Figure 1.

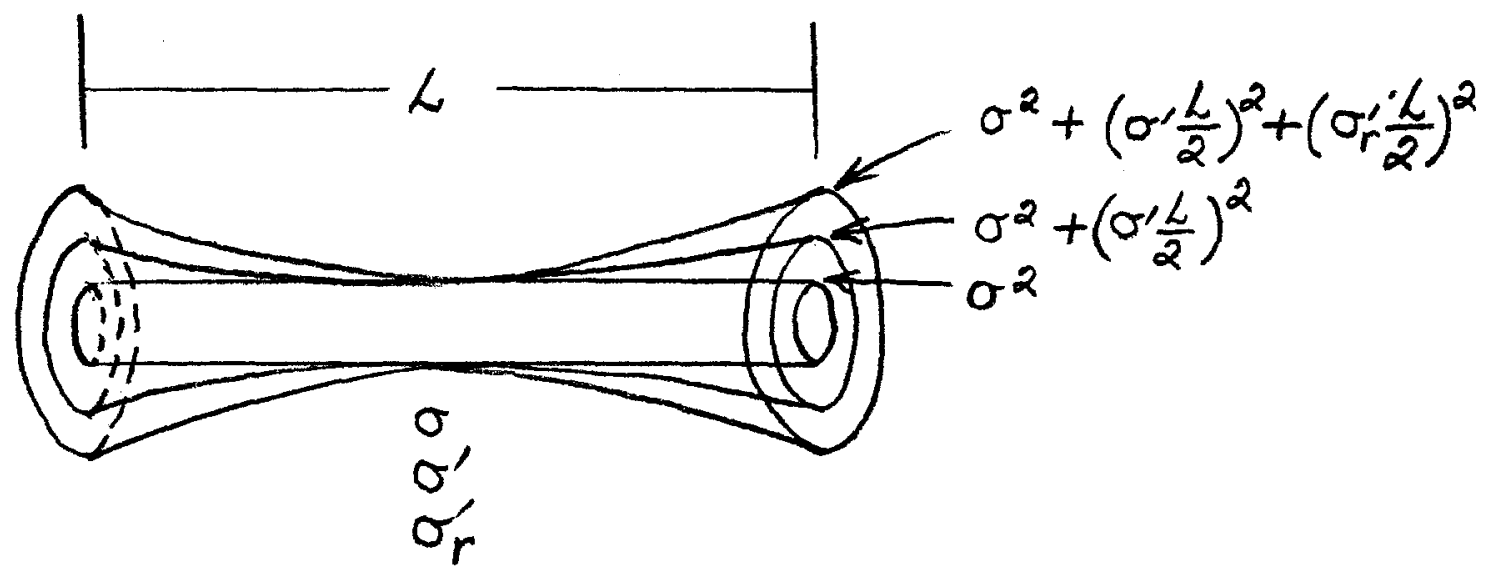

Figure 1 Apparent source size and divergence

B. Spectral Flux, Brightness, and Brilliance

These are defined as follows 


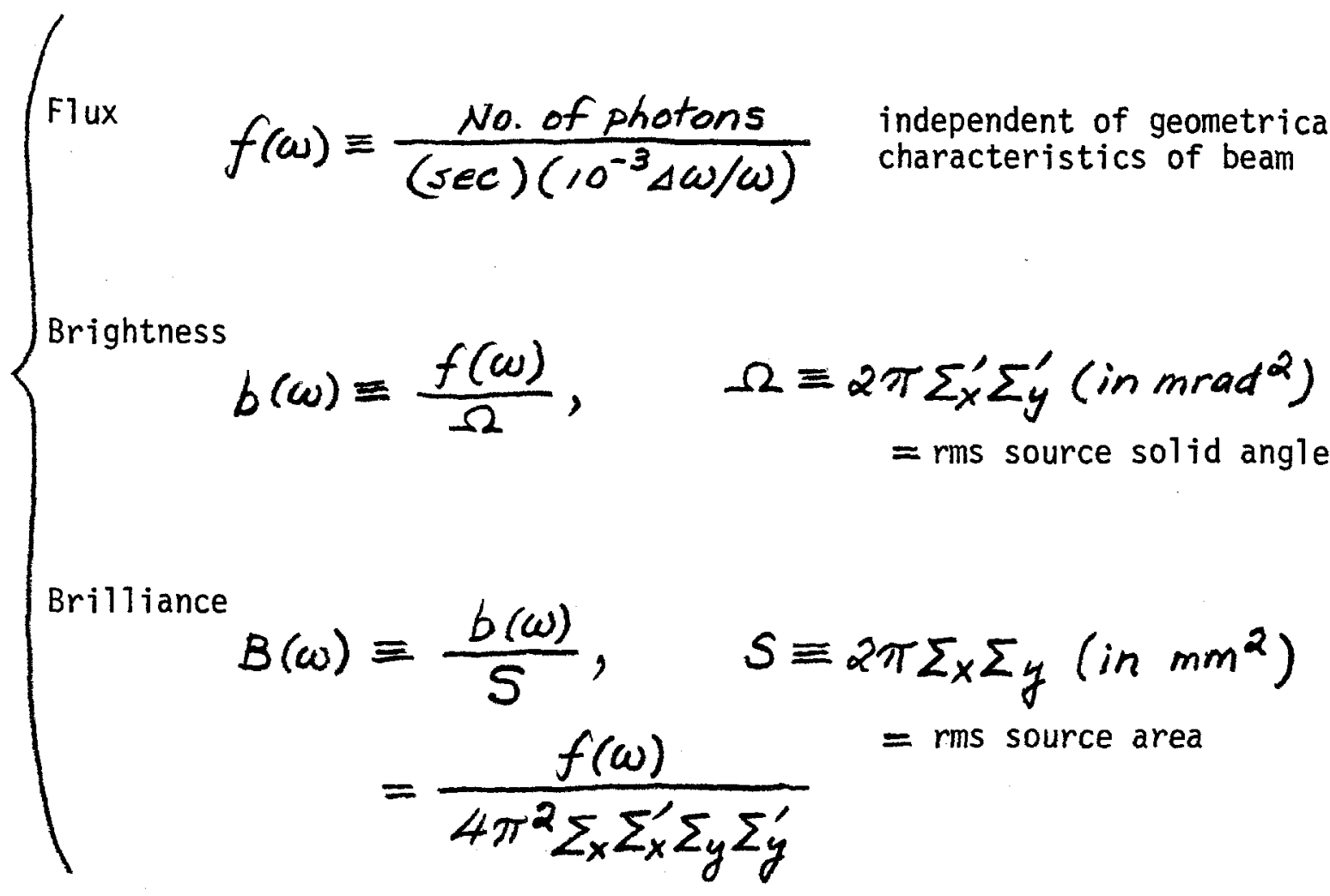

So defined the flux $f$ is independent of the geometrical characteristics of the electron beam. The dependence of the brilliance $B$ on the geometrical properties of the beam is only through the denominator

$$
S \Omega=4 \pi^{2} \Sigma_{x} \Sigma_{x}^{\prime} \Sigma_{y} \Sigma_{y}^{\prime}=\begin{aligned}
& \text { Apparent source } \\
& \text { phase-space volume }
\end{aligned}
$$

An approximate formula for $f$ was derived by D.F. Alferov, Yu. Bashmakov, and E.G. Bessonov (Eng. Trans]. Sov. Phys. Tech. Phys. Vo1. 18, p. 1336, 1974). They gave also the exact formula, but it is rather 
$-6-$

unwieldily to use. They derive first an intrinsic forward radiation intensity (assuming all electrons are on the same orbit)

$$
\left[\frac{d n\left(\omega_{\star}\right)}{d \Omega}\right]_{\theta=0}=\alpha N^{2} \gamma^{2} \frac{I}{\epsilon} \frac{\Delta \omega}{\omega} F_{\star}(K)
$$

where

$$
\alpha=\frac{1}{13 T}=\text { fine-structure content }
$$

and

$$
F_{t}(K)=\frac{4 k^{2} K^{2}}{\left(2+K^{2}\right)^{2}}\left[J_{\frac{k+1}{2}}\left(\frac{k K^{2}}{4+2 K^{2}}\right)-J_{\frac{k-1}{2}}\left(\frac{k K^{2}}{4+2 K^{2}}\right)\right]^{2}
$$

with $J$ being the Bessel function. To get the total flux $f$ we should integrate $\mathrm{dn} / \mathrm{d} \Omega$ over all solid angle. This is equivalent to multiplying the forward intensity $\left[\frac{d n}{d \Omega}\right]_{\theta=0}$ by the diffraction solid angle $2 \pi \sigma_{r}-2$, namely

$$
\begin{aligned}
f_{t}(\omega) & =2 \pi \sigma_{r}^{\prime 2}\left[\frac{d n(\omega)}{d \Omega}\right]_{\theta=0, \frac{\Delta \omega}{\omega}=10^{-3}} \\
& =\left(10^{-3}\right) \pi \propto N \frac{I}{e} Q_{t}
\end{aligned}
$$

where

$$
Q_{t}=4 u\left[J_{\frac{t+1}{2}}(u)-J_{\frac{t-1}{2}}(u)\right]^{2}
$$


$-7-$

with

$$
u \equiv \frac{k}{2} \frac{k^{2} / 2}{1+k^{2 / 2}}
$$

C. Maximizing the Brilliance $B(\omega)$

For this we want first to maximize the flux $f$. This is independently desirable for many types of use of the radiation. This has to do only with the design of the undulatory. Then, we want to minimize the source phase-space volume $S \Omega$. For this one has to tune for the desired electron beam size in the undulator.

1. Maximizing $f$

We note first that $K$ is related to the peak field $B_{0}$ by

$$
K=9.34 B_{0} l \quad\left(B_{0} l\right. \text { in kG m) }
$$

Since generally the total length $L$ is 1 imited we need to express $N$ in terms of $L$ by

$$
N=\frac{L}{l}=9.34 B_{0} L \frac{1}{K}
$$

and get for $f$

$$
f_{t}(\omega)=\left(9.34 \times 10^{-3}\right) \pi \alpha B_{0} \alpha \frac{I}{e} \frac{Q_{k}}{K}
$$

Thus, we see that for given values of $B_{0}, L$, and $I$ we want to maximize $Q_{k} / K$. The values of $Q_{k} / K$ are plotted as functions of $K$ for various values of $k$ in Figure 1. From these graphs we see that aside from $k=1$ and 3 the maxima are all rather flat. 
As an example, take the case of $k=1$ (the fundamental harmonic). The maximum value of $Q_{1} / K \cong 0.55$ occurs at $K \cong 1$. Equation (4) then gives

$$
\lambda_{1}=\frac{\ell}{2 \gamma^{2}}\left(1+\frac{k^{2}}{2}\right) \cong\left(2 \times 10^{-7}\right) \ell
$$

for $1 \mathrm{GeV}$ electrons. If one is interested in the VUV light of, say, $\lambda_{1}=10^{-8} \mathrm{~m}$, one gets $\ell=5 \mathrm{~cm}$. Equation (14) then gives $B_{0} \cong 2 \mathrm{kG}$. With a 100-cel1 ( $L=5 \mathrm{~m})$ undulator and an electron beam current of $500 \mathrm{~mA}$ one gets from Eq. (16) a total flux of $f \equiv 3.7 \times 10^{15} \mathrm{sec}^{-1}$.

2. Minimizing $S \Omega=4 \pi^{2} \cdot \Sigma_{x} \Sigma_{x}-\Sigma_{y} \Sigma_{y}^{\prime}$

For each $x$ or $y$ plane we have

$$
\Sigma^{2} \Sigma^{\prime 2}=\left(\sigma^{2}+\sigma^{\prime 2} \frac{L^{2}}{4}+\sigma_{r}^{\prime 2} \frac{L^{2}}{4}\right)\left(\sigma^{\prime 2}+\sigma_{r}^{\prime 2}\right)
$$

This can be simplified by noting that in most cases the beam has a waist at the mid-point of the undulator and that we can, therefore, write

$$
\sigma^{2}=\varepsilon \beta, \quad \sigma^{2}=\varepsilon / \beta, \quad \sigma_{r}^{\prime 2}=\lambda / L
$$

This gives

$$
\Sigma^{2} \Sigma^{2}=\varepsilon^{2}\left[1+\frac{\lambda}{\varepsilon} \frac{\beta}{L}+\frac{1}{4}\left(\frac{L}{\beta}+\frac{\lambda}{\varepsilon}\right)^{2}\right]
$$

Defining

$$
G \equiv\left[1+\frac{\lambda}{\varepsilon} \frac{\beta}{\alpha}+\frac{1}{4}\left(\frac{\alpha}{\beta}+\frac{\lambda}{\varepsilon}\right)^{2}\right]^{1 / 2}
$$

we can then write the source phase-space volume as 


$$
s \Omega=4 \pi^{2} \varepsilon_{x} \varepsilon_{y} G_{x} G_{y}
$$

The emittances $\varepsilon_{x}$ and $\varepsilon_{y}$ are determined by the design of the storage ring. One is therefore left only with the tuning of $B_{x}$ and $\beta_{y}$ to minimize $G_{x}$ and $G_{y}$. In Figure 2 we plot $G$ as a function of $B / L$ for various values of the parameter $\lambda / \varepsilon$. We now give an itemized discussion of these graphs.

a. The parameter $\lambda / \varepsilon$ has the following meaning. The contributions to the source size and the source divergence due to diffraction may be considered as $\sigma_{r}{ }^{\prime} \frac{L}{2}$ and $\sigma_{r}$ ' respectively. Hence we can define $\sigma_{r}{ }^{-2} \frac{L}{2}=\frac{\lambda}{2}$ as the diffraction contribution to the source phase-space volume (emittance). The parameter $\lambda / \varepsilon$ can therefore be interpreted as the ratio between the diffraction "emittance" and the beam emittance. When $\lambda / \varepsilon<2$ the beam dominates in determining the total source phase-space volume $S \Omega$, and when $\lambda / \varepsilon>2$ the diffraction dominates.

$$
\begin{aligned}
& \text { b. For the beam dominated case }\left(\frac{\lambda}{\varepsilon} \rightarrow 0\right) \text { Eq. (21) gives } \\
& G \cong\left(1+\frac{1}{4} \frac{L}{\beta}\right)^{1 / 2}
\end{aligned}
$$

In Figure 2 this is approached by the curve for $\lambda / \varepsilon=0.01$. The beam dominated limiting curve, furthermore, approaches the asymptotic value of $G=1$ as $B / L$ goes to infinity. For the diffraction dominated case $\left(\frac{\lambda}{\varepsilon} \rightarrow \infty\right)$ we have

$$
\begin{aligned}
G & \cong\left[\frac{1}{4}\left(\frac{\lambda}{\varepsilon}\right)^{2}+\frac{\lambda}{\varepsilon} \frac{\beta}{L}\right]^{1 / 2}=\frac{1}{2} \frac{\lambda}{\varepsilon}\left(1+\frac{4 \beta / L}{\lambda / \varepsilon}\right)^{1 / 2} \\
& \cong \frac{1}{2} \frac{\lambda}{\varepsilon}+\frac{\beta}{L}
\end{aligned}
$$


i.e. the curve is approximately a straight line starting at $\frac{1}{2} \frac{\lambda}{\varepsilon}$ at $\frac{B}{L}=0$ and increasing with $B / L$ at a unit slope. This gives

$$
\varepsilon G \cong \frac{\lambda}{2}+\frac{\varepsilon \beta}{L}=\frac{\lambda}{2}+\frac{\sigma^{2}}{L}
$$

where the second term is very small.

c. A simplified reasoning arising out of the second factor (the divergence) on the right-hand-side of Eq. (18) would conclude that the beam divergence should not be larger than the diffraction divergence, namely

$$
\sigma^{\prime 2}<\sigma_{r}^{\prime 2} \quad \text { or } \quad \frac{\varepsilon}{\beta}<\frac{\lambda}{L} \quad \text { or } \quad \frac{\beta}{\alpha}>\frac{\varepsilon}{\lambda}
$$

This is certainly true if one is considering the brightness $b$ which has only the solid angle $\Omega$ in the denominator. But for the brilliance with the denominator $S \Omega$ the situation is not so clear. A reduction in the beam divergence $\sigma^{\prime}$ (by an increase of $\beta$ ) is accompanied by an increase in the beam size $\sigma$. The graphs show that as $\beta / L$ decreases beyond a limit, $G$ does indeed rise sharply but the limit is rather insensitive to $\lambda / \varepsilon$. Over the 4-decade range of $\frac{\lambda}{\varepsilon}$ from 0.01 to 100 , the simple condition

$$
\frac{\beta}{2}>\frac{1}{2}
$$

does fairly well in ensuring that $S \Omega$ is no more than $-40 \%$ higher than the minimum.

$$
\text { Because } \varepsilon_{x} \text { and } \varepsilon_{y} \text { are very different in magnitude, }
$$
the two planes are unusually dominated by different factors and it is difficult 
to draw further general conclusions beyond the obvious statement that smaller beam emittances are better. For an example, we take $\varepsilon_{x}=2.6 \times 10^{-8} \mathrm{~m}$ and $\varepsilon_{y}=2.6 \times 10^{-10} \mathrm{~m}$ as in the TLS. If we are interested in $\lambda=10^{-8}$ (VUV) we have $\frac{\lambda}{\varepsilon_{x}} \sim 0.4$ and $\frac{\lambda}{\varepsilon_{y}} \sim 40$. In the $y$-plane the $\frac{\lambda}{\varepsilon}=40$ curve gives a near minimum $G_{y} \cong 22$ at $\beta_{y} / L \cong 0.3$ or $\beta_{y}=1.5 \mathrm{~m}$ for $L=5 \mathrm{~m}$. The tuning curve of $T L S$ gives for this $\beta_{y}$ value a $\beta_{x}=9.5 \mathrm{~m}$. The $\frac{\lambda}{\varepsilon}=0.4$ curve gives $G_{x} \cong 1.4$ (minimum is 1.35) at $\beta_{x} / L=1.9$. Thus al together we have

$$
\begin{aligned}
S \Omega & =4 \pi^{2}\left(2.6 \times 10^{-8} \mathrm{~m}\right)\left(2.6 \times 10^{-10} \mathrm{~m}\right) \times 1.4 \times 22 \\
& =8.2 \times 10^{-15} \mathrm{~m}^{2}=8.2 \times 10^{-3} \mathrm{~mm}^{2} \mathrm{mrad}^{2}
\end{aligned}
$$

Together with the maximized flux $f=3.7 \times 10^{15} \mathrm{sec}^{-1}$ this gives a maximized brilliance of

$$
\begin{aligned}
B & =\frac{3.7 \times 10^{15}}{8.2 \times 10^{-3}} \sec ^{-1} \mathrm{~mm}^{-2} \operatorname{mrad}^{-2} \\
& =0.45 \times 10^{18} \mathrm{sec}^{-1} \mathrm{~mm}^{-2} \operatorname{mrad}^{-2}\left(10^{-3} \mathrm{A \omega} / \omega\right)^{-1}
\end{aligned}
$$

The undulator parameters are

$$
l=5 \mathrm{~cm}, \quad B_{0}=2 \mathrm{kG}, \quad N=100, \quad L=5 \mathrm{~m}
$$

and the tuning parameters are

$$
\beta_{x}=9.5 \mathrm{~m}, \quad \beta_{y}=1.5 \mathrm{~m}
$$

Figures 1 and 2 are computed and plotted by $Y$. Cho of Argonne. His able assistance is gratefully acknowledged. 


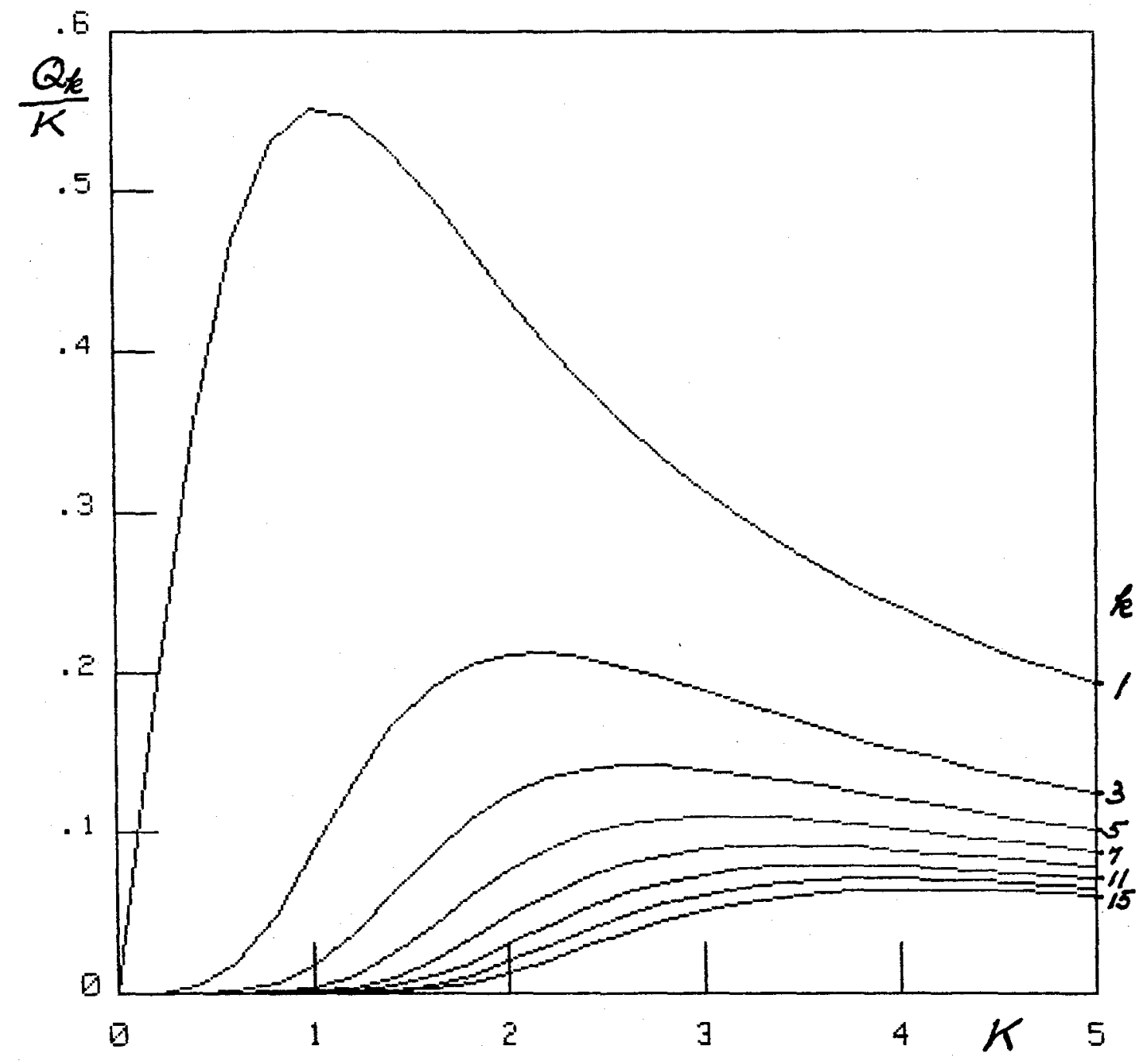

Figure 1 $Q_{k} / K$ plotted as functions of $K$ for $k=1,3,5,7,9,11,13,15$ 


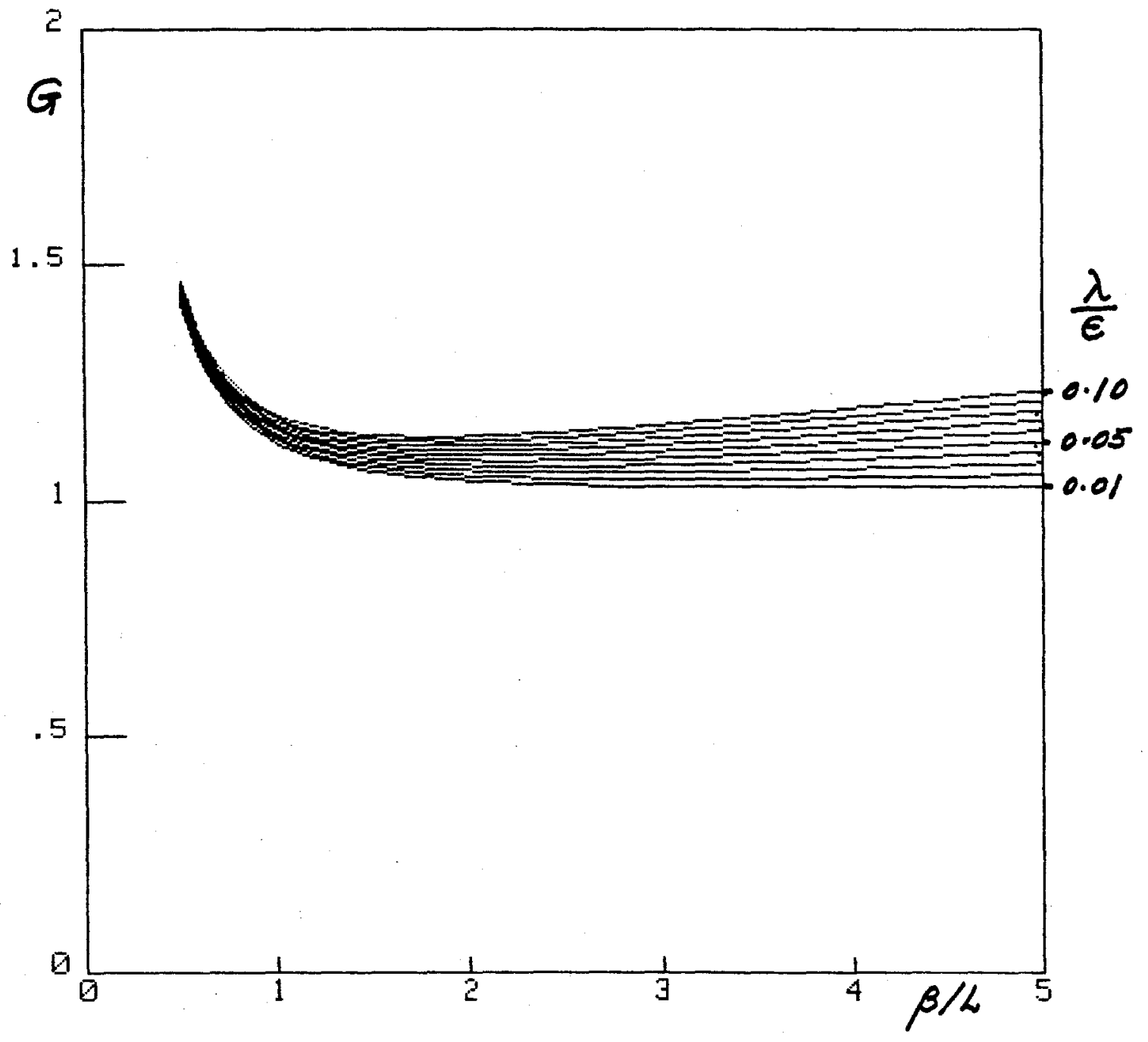

Figure $2 a, b, c, d . \quad G$ plotted as functions of $B / L$ for the ranges of value of $\lambda / \varepsilon$ :
a. $0.01-0.10$
b. $0.10-1.0$
c. $1.0-10$
d. $10-100$ 


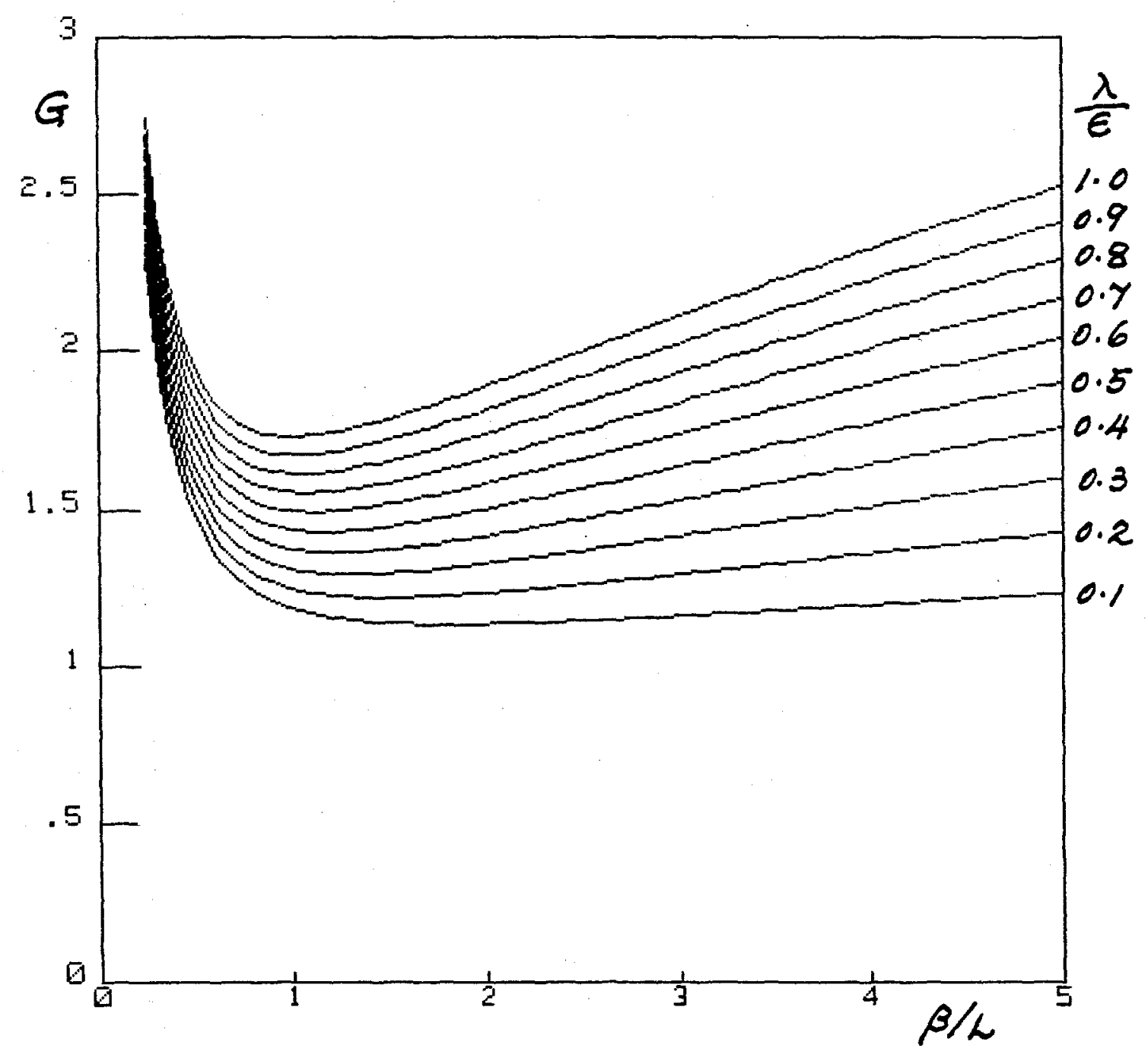

Figure 2b 


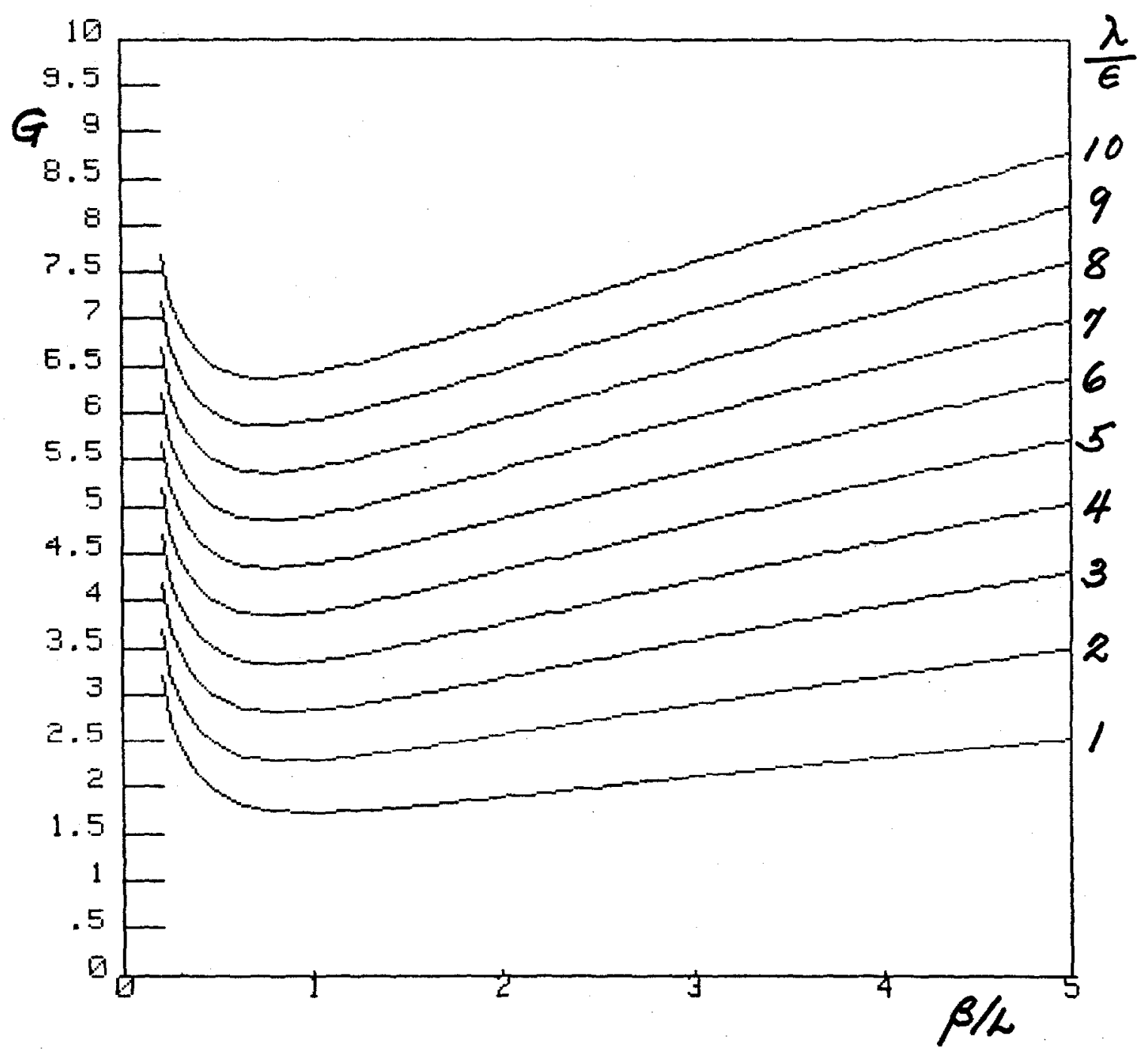

Figure 2c 


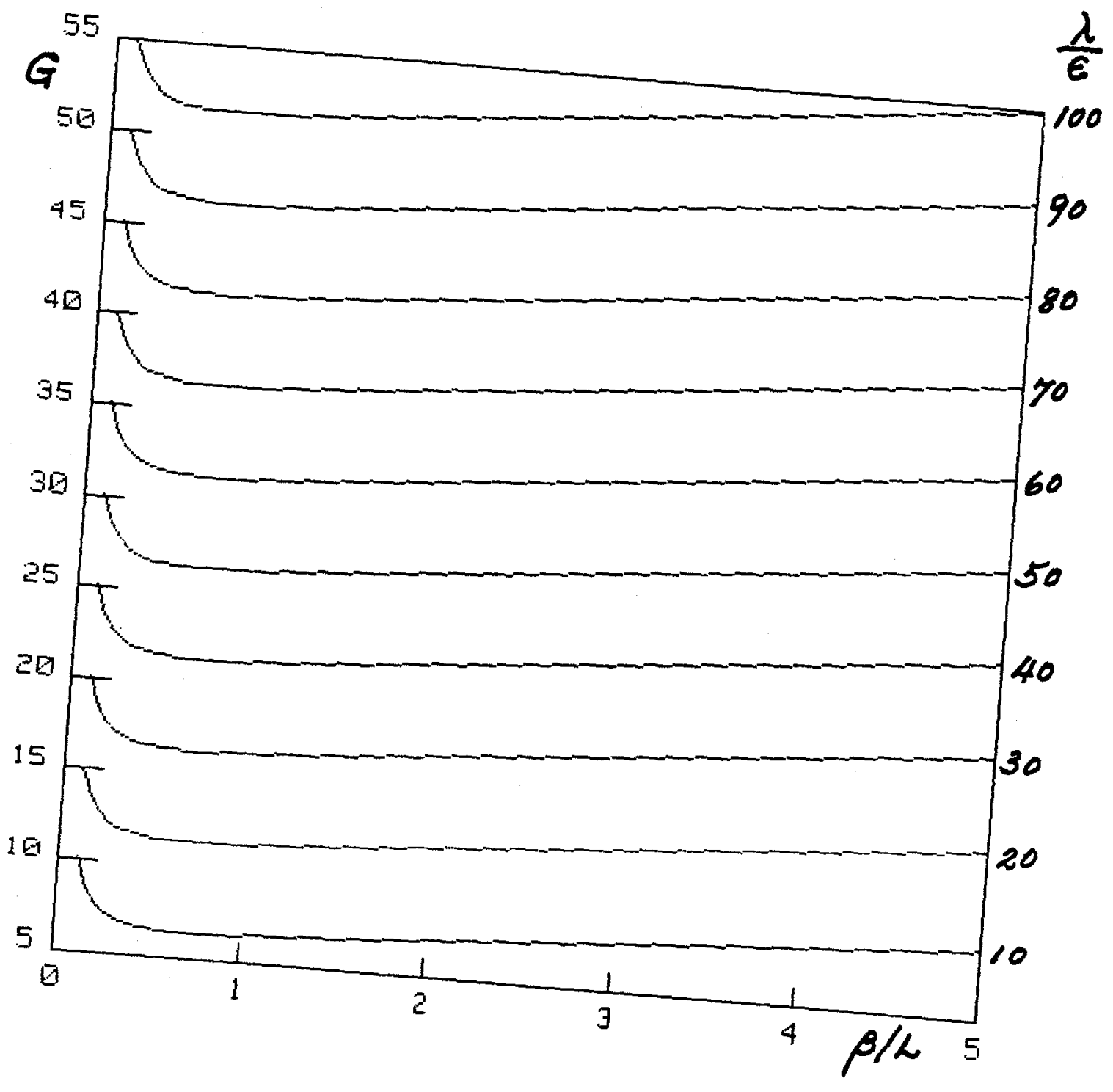

Figure 2d 